\title{
Associations of maternal PLA2G4C and PLA2G4D polymorphisms with the risk of spontaneous preterm birth in a Chinese population
}

\author{
GUANG-JIAN LIU ${ }^{1 *}$, JIAN-RONG HE $^{1 *}$, YA-SHU KUANG ${ }^{1 *}$, XUE-JIAO FAN ${ }^{1}$, WEI-DONG LI ${ }^{1}$, JIN-HUA LU $^{1}$, \\ XIAO-YAN XIA ${ }^{1}$, XIAO-DAN LIU ${ }^{1}$, NIAN-NIAN CHEN ${ }^{1}$, WEI-BI MAI ${ }^{2}$, HUI-MIN XIA ${ }^{1}$ and XIU QIU ${ }^{1}$ \\ ${ }^{1}$ Division of Birth Cohort Study, and ${ }^{2}$ Department of Obstetrics and Gynecology, Guangzhou Women and \\ Children's Medical Center, Guangzhou Medical University, Guangzhou, Guangdong 510623, P.R. China
}

Received February 23, 2016; Accepted February 22, 2017

DOI: $10.3892 / \mathrm{mmr} .2017 .6475$

\begin{abstract}
Preterm birth is the leading cause of mortality and morbidity in infants. Its etiology is multifactorial with genes and immune homeostasis. The authors investigated whether prostaglandin (PG) synthesis related single nucleotide polymorphisms (SNPs) PLA2G4C rs1366442 and PLA2G4D rs4924618 were associated with the risk of spontaneous preterm birth (SPTB) in a Chinese population of 114 cases of SPTB and 250 controls of term delivery. The risk associations were determined by odds ratios (ORs) and their $95 \%$ confidence intervals (CIs) calculated using multivariate logistic regression. Homology modeling was performed to elucidate potential mechanism of the SNP function. The maternal AT/ TT genotype of PLA2G4D rs4924618 was associated with a reduced risk of SPTB (OR, 0.61; 95\% CI, 0.37-0.99), while no significant association between PLA2G4C rs1366442 and SPTB risk was identified. Structure and sequence analysis revealed that the amino acid substitution introduced by this SNP located at the conserved central core of the catalytic domain of cytosolic phospholipase A $2 \delta$ and was close to the active site. These findings suggested that the polymorphism of PLA2G4D rs4924618 may have a protective influence on the SPTB susceptibility in a Chinese population, supporting a
\end{abstract}

Correspondence to: Dr Xiu Qiu or Professor Hui-Min Xia, Division of Birth Cohort Study, Guangzhou Women and Children's Medical Center, 9 Jinsui Road, Guangzhou, Guangdong 510623, P.R. China

E-mail: qxiu0161@163.com

E-mail: huimin.xia876001@gmail.com

${ }^{*}$ Contributed equally

Abbreviations: SNP, single nucleotide polymorphism; SPTB, spontaneous preterm birth; PGs, prostaglandins; cPLA2, cytosolic phospholipase A2; GDM, gestational diabetes mellitus; GWCMC, Guangzhou Women and Children's Medical Center

Key words: PLA2G4C, PLA2G4D, polymorphism, spontaneous preterm birth role for genetics in the association between PG synthesis and preterm birth.

\section{Introduction}

Preterm birth, defined as birth before 37 weeks of gestation, accounts for $75 \%$ of infant mortality worldwide and is the leading cause of the long-term morbidity, such as neurodevelopmental impairments, respiratory and gastrointestinal complications $(1,2)$. Between 65 and $75 \%$ of preterm births follow spontaneous preterm birth (SPTB), but the precise pathologic mechanisms remain to be elucidated due to their complexity (3). Previous studies analyzing the recurrence of SPTB across generations and between siblings suggested that genetic factors are likely contributors to variations in the length of gestation with heritability estimates in the range of $27-36 \%(4,5)$. Numerous candidate gene association studies have explored the role of single nucleotide polymorphisms (SNPs) in the development of SPTB and differences in the prevalence across populations (6-11). Meanwhile, a publicly available online database of genetic association data on preterm birth has been established to keep track of the evolving evidence base of genetic factors in preterm birth (12).

Prostaglandins (PGs) serve an important role in the processes of term and preterm labor, contributing to uterine contractility, membrane rupture and cervical ripening $(13,14)$. The rate-limiting step in PG biosynthesis is catalyzed by phospholipase A2 (PLA2) enzymes, which comprise $>20$ members $(15,16)$. One member of PLA2 family, group IVC PLA2 (PLA2G4C), has been reported to be expressed in uterus and regulate uterine PG production by its expression and activity (17). Previously, polymorphisms in this gene were identified to be associated with the risk of preterm birth in a case-control study with US Hispanic, White and Black mothers (18). It remains unclear whether these polymorphisms also confer SPTB susceptibility in Asians.

In addition to PLA2G4C, it should be noted that another member of PLA2 family, group IVD PLA2 (PLA2G4D) was demonstrated to be uniquely expressed in the stratified squamous epithelium of the cervix and involved in $\mathrm{PG}$ regulation (19). PLA2G4D encodes for a protein of $\sim 90 \mathrm{kDa}$ 
with significant homology with known cytosolic PLA2 proteins in the catalytic domains and has been implicated to serve a role in many diseases, such as schizophrenia, autism and psoriatic skin (19-21). However, to date, no published data have examined the association of the polymorphisms of PLA2G4D with SPTB.

With these in mind, the authors hypothesized that PLA2G4C and PLA2G4D may be potential candidates for SPTB susceptibility. The associations of maternal PLA2G4C rs1366442 and PLA2G4D rs4924618 polymorphisms were investigated with the risk of SPTB in a case-control study with a Chinese population. Moreover, bioinformatics tools were used to elucidate potential mechanism of the SNP function.

\section{Materials and methods}

Subjects. The present study is a nested case-control study, embedded in a prospective study from the Guangzhou Women and Children's Medical Center (GWCMC) that was designed to investigate the health consequences of genetic and environmental factors on pregnancy outcomes and offspring. Pregnant women who attended the routine oral glucose tolerance test were invited to participate in the study at 24-28 weeks of gestation, if they met the following inclusion criteria: $\geq 18$ years old, Chinese and intend to deliver at GWCMC. From April 2013 to March 2014, 3597 (69.0\% of those eligible) pregnant women were enrolled to this prospective study and donated their blood samples.

Of those who gave singleton live birth, 174 women had preterm deliveries ( $<37$ weeks of gestation). Women with medically indicated preterm delivery $(\mathrm{n}=53)$, in vitro fertilization $(n=5)$, fetal anomaly $(n=1)$ or uterine malformation $(n=1)$ were excluded, resulting in 114 women having a spontaneous preterm birth (42 preterm labor and 72 preterm premature rupture of membranes) in the present study as the case group. In addition, 250 pregnant women, who delivered singleton term babies (38-41 weeks of gestation) and had no preeclampsia, eclampsia, placental abruption, placenta previa, haemolysis, elevated liver enzymes or low platelet count syndrome, were selected and included as the control group. Controls were frequency-matched to the cases by age within 3 years. The study was approved by the Institutional Review Board of GWCMC. Informed consent was obtained from all participants.

SNP selection. To select SNPs, the authors conducted a systematic literature review, assessing the evidence for the associations of PLA2G4C and PLA2G4D with SPTB. For the PLA2G4C gene, four SNPs had been reported to influence preterm birth risk in the US Hispanic or White populations (18). However, three of them (rs8110925, rs2307276 and rs11564620) were excluded due to the minor allele frequencies (MAF) reported in HapMap (http://hapmap.ncbi.nlm. nih.gov/) were $<10 \%$ for Chinese subjects. Only rs1366442 in the intron region with a MAF of $13 \%$ was chosen to ensure that an adequate number of individuals within the population would be carriers of the minor allele. For the PLA2G4D gene, published reports on polymorphisms of this gene and diseases are rare. The authors chose rs 4924618 based on the following two reasons: i) a weak association between this SNP and schizophrenia was observed in a Chinese population (20), while schizophrenia was related to a higher risk of preterm birth $(22,23)$; ii) this SNP is a non-synonymous SNP that is likely to alter the protein folding structure, suggesting that this SNP may have a functional effect.

Genotyping. Blood samples were stored at $-80^{\circ} \mathrm{C}$ until laboratory use. Genomic DNA was extracted from cells isolated from $2 \mathrm{ml}$ of blood samples using the Qiagen DNA Blood Mini kit (Qiagen, Inc., Valencia, CA, USA) according to the manufacturer's instructions. Genotyping for PLA2G4C rs1366442 and PLA2G4D rs4924618 was performed by using a MassARRAY system (Sequenom, San Diego, CA, USA). A total of 20 samples (5.5\% of total) were tested in duplicates to determine the quality of genotyping, and the concordance rates were $100 \%$. DNA from $4(1.1 \%)$ and $5(1.4 \%)$ samples failed to be genotyped for PLA2G4C rs1366442 and PLA2G4D rs4924618.

Covariates. Information on maternal age, educational level, smoking status, maternal height and pre-pregnancy weight, parity and previous history of preterm delivery were collected by using face to face questionnaire. In addition, diagnosis of gestational diabetes mellitus (GDM), infant's gestational age, birth weight and sex, were abstracted from medical records. Gestational age was evaluated based on the ultrasound examination in the first or second trimester. Pre-pregnancy body mass index (BMI; $\mathrm{kg} / \mathrm{m}^{2}$ ) was calculated as the ratio of pre-pregnancy weight $(\mathrm{kg})$ to squared height $(\mathrm{m})$. Since the prevalence of previous history of preterm delivery and smoking exposure were quite low ( $\leq 1 \%$ in the control group), the authors did not include these variables for further analysis.

Statistical analysis. The differences between the case and control groups were evaluated by using the chi-squared test or Fisher's exact test (for categorical variables) and Student's t-test (for continuous variables). The Hardy-Weinberg equilibrium for PLA2G4C rs1366442 and PLA2G4D rs4924618 was examined by a goodness-of-fit chi-squared test among the control group. The multivariate logistic regression was used to investigate the relationship between genotypes and the risk of preterm birth, adjusting for maternal age, educational level (high school or below vs. college or above), pre-pregnancy BMI, parity (primiparous vs. multiparous), GDM status (yes vs. no) and infant's sex. The odds ratios (ORs) and the corresponding 95\% confidence intervals (CIs) were calculated. Stratified analysis was performed to examine whether the effects of genotypes with the risk of preterm birth varied across different status of maternal age ( $<30$ vs. $\geq 30$ ), GDM status (no vs. yes) and infant's sex (female vs. male). The multiplicative interaction between genotypes and participants' characteristics were evaluated in the logistic regression models.

A two-tailed $\mathrm{P}<0.05$ was considered statistically significant. All statistical analysis was performed using SAS statistical software (version, 9.3; SAS Institute, Cary, NC, USA).

cPLA2 structure model. The structure of cPLA2 $\delta$ catalytic domain was constructed through homology modeling. Sequences of four cytosolic phospholipase A2 (cPLA2) $(\alpha, \beta, \gamma$ and $\delta$ ) were obtained from UniProt (www.uniprot. 
Table I. Characteristics of participants.

\begin{tabular}{|c|c|c|c|}
\hline \multicolumn{4}{|l|}{ A, Mother } \\
\hline Characteristics & Cases, n (\%) & Controls, n (\%) & P-value \\
\hline \multicolumn{4}{|l|}{ Age (years) } \\
\hline$<30$ & $55(48.3)$ & $127(50.8)$ & \\
\hline$\geq 30$ & $59(51.8)$ & $123(49.2)$ & 0.65 \\
\hline Mean (SD) & $29.8(3.5)$ & $29.8(3.5)$ & 0.97 \\
\hline \multicolumn{4}{|l|}{ Educational level } \\
\hline High middle school or below & $12(10.5)$ & $36(14.4)$ & 0.31 \\
\hline College or above & $102(89.5)$ & $214(85.6)$ & \\
\hline \multicolumn{4}{|l|}{ Marital Status } \\
\hline Unmarried & $0(0.0)$ & $2(0.8)$ & $>0.99^{\#}$ \\
\hline Married & $114(100)$ & $248(99.2)$ & \\
\hline \multicolumn{4}{|l|}{ Parity } \\
\hline Primiparous & $92(80.7)$ & $225(90.0)$ & 0.01 \\
\hline Multiparous & $22(19.3)$ & $25(10.0)$ & \\
\hline \multicolumn{4}{|l|}{ History of abortion } \\
\hline Yes & $73(76.8)$ & $149(73.0)$ & 0.48 \\
\hline No & $22(23.2)$ & $55(27.0)$ & \\
\hline No data & 19 & 46 & \\
\hline \multicolumn{4}{|c|}{ Previous history of preterm delivery } \\
\hline No & $94(100.0)$ & $205(99.0)$ & $>0.99^{\#}$ \\
\hline Yes & $0(0.0)$ & $2(1.0)$ & \\
\hline No data & 20 & 43 & \\
\hline \multicolumn{4}{|l|}{ Pre-pregnancy BMI } \\
\hline$<20$ & $74(64.9)$ & $169(67.6)$ & 0.61 \\
\hline$\geq 20$ & $40(35.1)$ & $81(32.4)$ & \\
\hline Mean (SD) & $20.0(2.1)$ & $19.8(2.1)$ & 0.53 \\
\hline \multicolumn{4}{|l|}{ Smoking } \\
\hline No & $67(100.0)$ & $152(99.4)$ & $>0.99^{\#}$ \\
\hline Yes & $0(0.0)$ & $1(0.7)$ & \\
\hline No data & 47 & 97 & \\
\hline \multicolumn{4}{|l|}{ GDM } \\
\hline No & $88(77.2)$ & $201(80.4)$ & 0.48 \\
\hline Yes & $26(22.8)$ & $49(19.6)$ & \\
\hline
\end{tabular}

B, Newborn

\begin{tabular}{|c|c|c|c|}
\hline Characteristics & Cases, n $(\%)$ & Controls, n (\%) & P-value \\
\hline \multicolumn{4}{|l|}{ Sex } \\
\hline Female & $46(40.4)$ & $145(58.0)$ & \\
\hline Male & $68(59.7)$ & $105(42.0)$ & 0.002 \\
\hline Birth weight (g), median (25th, 75th) & $2660(2300,2880)$ & $3280(3030,3540)$ & $<0.001$ \\
\hline Gestational age (weeks), median (25th, 75th) & $36(35,36)$ & $39(39,40)$ & $<0.001$ \\
\hline
\end{tabular}

P-values indicate comparisons between the different subgroups of each characteristic. "Fisher exact test. SD, standard deviation; BMI, body mass index; GDM, gestational diabetes mellitus.

org). To check the identity and homology, sequences of cPLA2 catalytic domains were compared by BLAST using Basic
Local Alignment Search Tool (http://blast.ncbi.nlm.nih.gov/ Blast.cgi) and ClustalW (https://www.ebi.ac.uk/Tools/msa/ 
Table II. Associations of genetic variants with the risk of SPTB.

\begin{tabular}{|c|c|c|c|c|}
\hline Genotype & Controls, n (\%) & Cases, n (\%) & OR $(95 \% \mathrm{CI})^{\mathrm{a}}$ & OR $(95 \% \mathrm{CI})^{\mathrm{b}}$ \\
\hline \multicolumn{5}{|c|}{ PLA2G4C rs1366442 } \\
\hline $\mathrm{CC}$ & $159(64.4)$ & $76(67.3)$ & 1.00 (reference) & 1.00 (reference) \\
\hline $\mathrm{CA}$ & $80(32.4)$ & $34(30.1)$ & $0.89(0.55,1.45)$ & $0.90(0.54,1.49)$ \\
\hline $\mathrm{AA}$ & $8(3.2)$ & $3(2.7)$ & $0.79(0.2,3.04)$ & $1.06(0.26,4.24)$ \\
\hline $\mathrm{CA} / \mathrm{AA}$ & $88(35.6)$ & $37(32.7)$ & $0.88(0.55,1.41)$ & $0.91(0.56,1.49)$ \\
\hline \multicolumn{5}{|c|}{ PLA2G4D rs4924618 } \\
\hline AA & $66(26.9)$ & $42(36.8)$ & 1.00 (reference) & 1.00 (reference) \\
\hline $\mathrm{AT}$ & $133(54.3)$ & $57(50.0)$ & $0.67(0.41,1.11)$ & $0.65(0.38,1.09)$ \\
\hline TT & $46(18.8)$ & $15(13.2)$ & $0.51(0.26,1.03)$ & $0.50(0.24,1.02)$ \\
\hline $\mathrm{AT} / \mathrm{TT}$ & $179(73.1)$ & $72(63.2)$ & $0.63(0.39,1.02)$ & $0.61(0.37,0.99)$ \\
\hline
\end{tabular}

OR and $95 \% \mathrm{CI}$ are presented relative to the reference group in each section. ${ }^{\mathrm{a}} \mathrm{Crude}$ OR and $95 \% \mathrm{CI} .{ }^{\mathrm{b}}$ Adjusted for maternal age, educational level, parity, pre-pregnancy BMI, status of GDM and infant sex. SPTB, spontaneous preterm birth; OR, Odds ratio; CI, confidence interval; BMI, body mass index; GDM, gestational diabetes mellitus.

Table III. Stratified analysis for associations between PLA2G4C and PLA2G4D variants and the risk of SPTB.

\begin{tabular}{|c|c|c|c|c|c|c|}
\hline \multirow[b]{2}{*}{ Stratified variables } & \multicolumn{3}{|c|}{ PLA2G4C rs1366442 } & \multicolumn{3}{|c|}{ PLA2G4D rs4924618 } \\
\hline & Genotype & Case/Control, n & OR $(95 \% \mathrm{CI})^{\mathrm{a}}$ & Genotype & Case/Control, n & OR $(95 \% \mathrm{CI})^{\mathrm{a}}$ \\
\hline \multicolumn{7}{|l|}{ Age } \\
\hline \multirow[t]{2}{*}{$<30$} & $\mathrm{CC}$ & $34 / 83$ & 1.00 (reference) & AA & $16 / 35$ & 1.00 (reference) \\
\hline & CA/AA & $20 / 43$ & $1.13(0.56,2.28)$ & $\mathrm{AT} / \mathrm{TT}$ & $39 / 89$ & $0.83(0.39,1.8)$ \\
\hline \multirow[t]{2}{*}{$\geq 30$} & $\mathrm{CC}$ & $42 / 76$ & 1.00 (reference) & AA & $26 / 31$ & 1.00 (reference) \\
\hline & CA/AA & $17 / 45$ & $0.75(0.37,1.51)$ & AT/TT & $33 / 90$ & $0.42(0.21,0.83)$ \\
\hline P-value for interaction & & & 0.33 & & & 0.10 \\
\hline \multicolumn{7}{|l|}{ GDM } \\
\hline \multirow[t]{2}{*}{ No } & $\mathrm{CC}$ & $60 / 132$ & 1.00 (reference) & AA & $32 / 52$ & 1.00 (reference) \\
\hline & CA/AA & $27 / 66$ & $1.02(0.58,1.79)$ & AT/TT & $56 / 144$ & $0.59(0.34,1.04)$ \\
\hline \multirow[t]{2}{*}{ Yes } & $\mathrm{CC}$ & $16 / 27$ & 1.00 (reference) & AA & $10 / 14$ & 1.00 (reference) \\
\hline & CA/AA & $10 / 22$ & $0.50(0.17,1.52)$ & AT/TT & $16 / 35$ & $0.68(0.22,2.08)$ \\
\hline P-value for interaction & & & 0.43 & & & 0.06 \\
\hline \multicolumn{7}{|l|}{ Sex } \\
\hline \multirow[t]{2}{*}{ Female } & $\mathrm{CC}$ & $46 / 71$ & 1.00 (reference) & $\mathrm{AA}$ & $27 / 27$ & 1.00 (reference) \\
\hline & $\mathrm{CA} / \mathrm{AA}$ & $21 / 31$ & $1.10(0.55,2.21)$ & AT/TT & $41 / 75$ & $0.47(0.23,0.94)$ \\
\hline \multirow[t]{2}{*}{ Male } & $\mathrm{CC}$ & $30 / 88$ & 1.00 (reference) & $\mathrm{AA}$ & $15 / 39$ & 1.00 (reference) \\
\hline & CA/AA & $16 / 57$ & $0.81(0.40,1.64)$ & AT/TT & $31 / 104$ & $0.76(0.36,1.59)$ \\
\hline $\mathrm{P}$-value for interaction & & & 0.67 & & & 0.31 \\
\hline
\end{tabular}

OR and 95\% CI are presented relative to the reference group in each section. ${ }^{a}$ Adjusted for maternal age, educational level, parity, pre-pregnancy BMI, status of GDM and infant sex. OR, Odds ratio; CI, confidence interval; BMI, body mass index; GDM, gestational diabetes mellitus.

clustalw2/). cPLA2 $\delta$ catalytic domain is localized between amino acids 273 and 818 and the cPLA2 $\alpha$ (Protein Data Bank ID: 1CJY) was chosen as a template. The SWISS-model workspace (https://swissmodel.expasy.org/; Swiss Institute of Bioinformatics, Basel, Switzerland) was used to construct the homology model of cPLA $2 \delta$ to obtain a structural insight of the protein and to locate the SNP-related amino acid in the protein. Manual alignment of cPLA2 $\delta$ to the template was exported in FASTA format to the Swiss-Model Server (24). The homology model was then validated by the Ramachandran plot plugin in VMD 1.9.2 (http://www. ks.uiuc.edu/; Theoretical and Computational Biophysics Group, NIH Center for Macromolecular Moedling and Bioinformatics, at the Beckman Institute, University of Illinois 
A

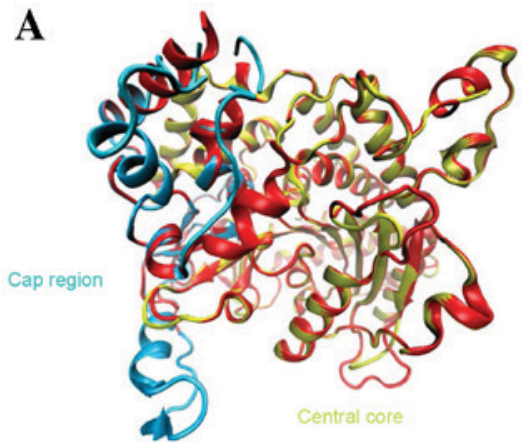

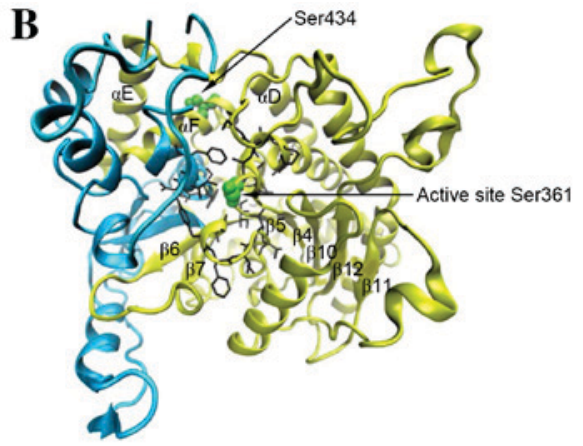

Figure 1. Tertiary structure of cPLA2 $\delta$. (A) Predictive 3D model aligned to the template (red, template; yellow and cyan, model). (B) Location of the mutation site and active site in cPLA2 $\delta$. The central core is presented in yellow, with the secondary structure elements labeled according to the crystal structure of cPLA2 $\alpha$. The cap region is colored blue. The mutation site Ser434 and active site Ser361 are displayed as green spheres. Side chains of residues constituting the active site funnel are displayed as colored sticks with black type. This image was made with VMD software support. VMD is developed with NIH support by the Theoretical and Computational Biophysics group at the Beckman Institute, University of Illinois at Urbana-Champaign (Champaign, IL, USA). cPLA2, cytosolic phospholipase A2.

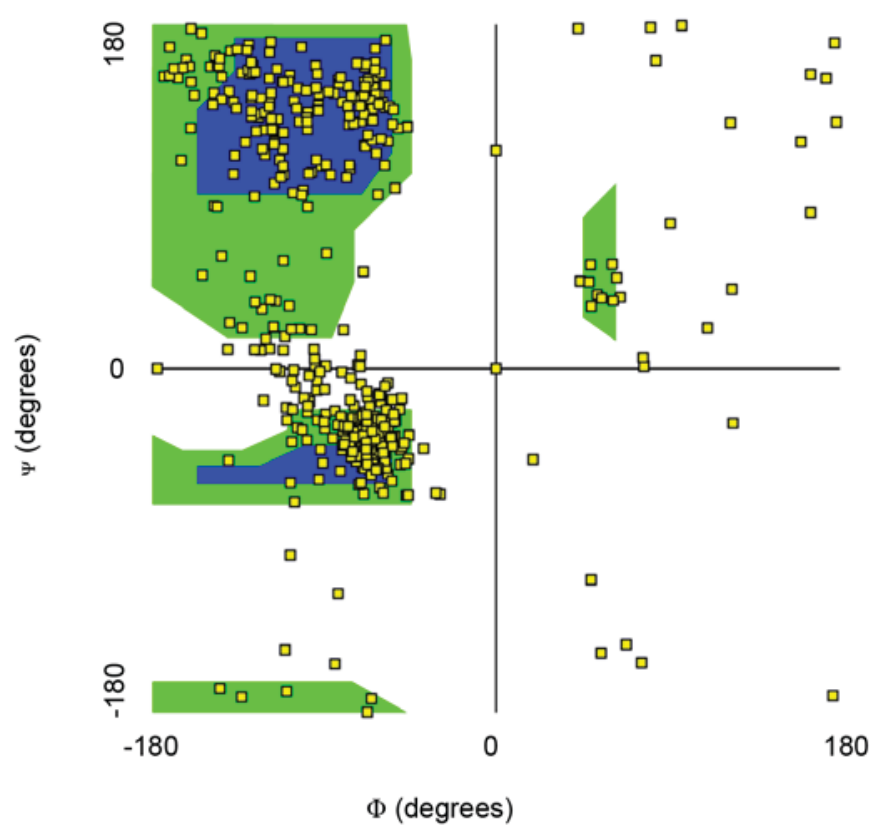

Figure 2. Ramachandran plot of a structural model of cPLA2 $\delta$. The most energetically allowed regions of Ramachandran space is colored blue and the allowed regions are colored green. This image was made with VMD software support. VMD is developed with NIH support by the Theoretical and Computational Biophysics group at the Beckman Institute, University of Illinois at Urbana-Champaign (Champaign, IL, USA). cPLA2, cytosolic phospholipase A2.

at Urbana-Champaign, Champaign, IL, USA) to determine stereochemical aspects along with main chain and side chain parameters (25).

\section{Results}

Demographic characteristics. The baseline characteristics of the studied population, including 114 SPTB cases and 250 controls, are presented in Table I. No significant differences were observed in the distributions of maternal age, educational level, history of abortion, history of preterm delivery, pre-pregnancy BMI, smoking status and GDM status between the cases and controls $(\mathrm{P}>0.05)$. As expected, gestational age at delivery $(\mathrm{P}<0.001)$ and birth weight $(\mathrm{P}<0.001)$ were significantly lower in the case group, when compared with those in the control group, respectively. In addition, cases were more likely to be multiparous $(\mathrm{P}=0.01)$ and neonates from the case group had higher percentage of boys $(\mathrm{P}=0.001)$.

Association of maternal PLA2G4C, PLA2G4D polymorphism with the risk of SPTB. Genotype distributions of the two selected SNPs in cases and controls are summarized in Table II. There was no deviation from the Hardy-Weinberg equilibrium observed among the controls $(\mathrm{P}=0.94$ for $\mathrm{rs} 1366442$ and $\mathrm{P}=0.58$ for rs4924618). Logistic regression analysis was performed to assess the effects of each SNP on SPTB risk. In the multivariate analysis, compared to the homozygous genotype GG, the TT genotypes of PLA2G4D rs4924618 was associated with a marginally reduced risk of SPTB (adjusted OR and 95\% CI, 0.50 [0.24-1.02]), and the AT/TT (dominant model) genotypes was associated with a reduced risk of SPTB (adjusted OR, 0.61 [0.37-0.99]). No significant association was 


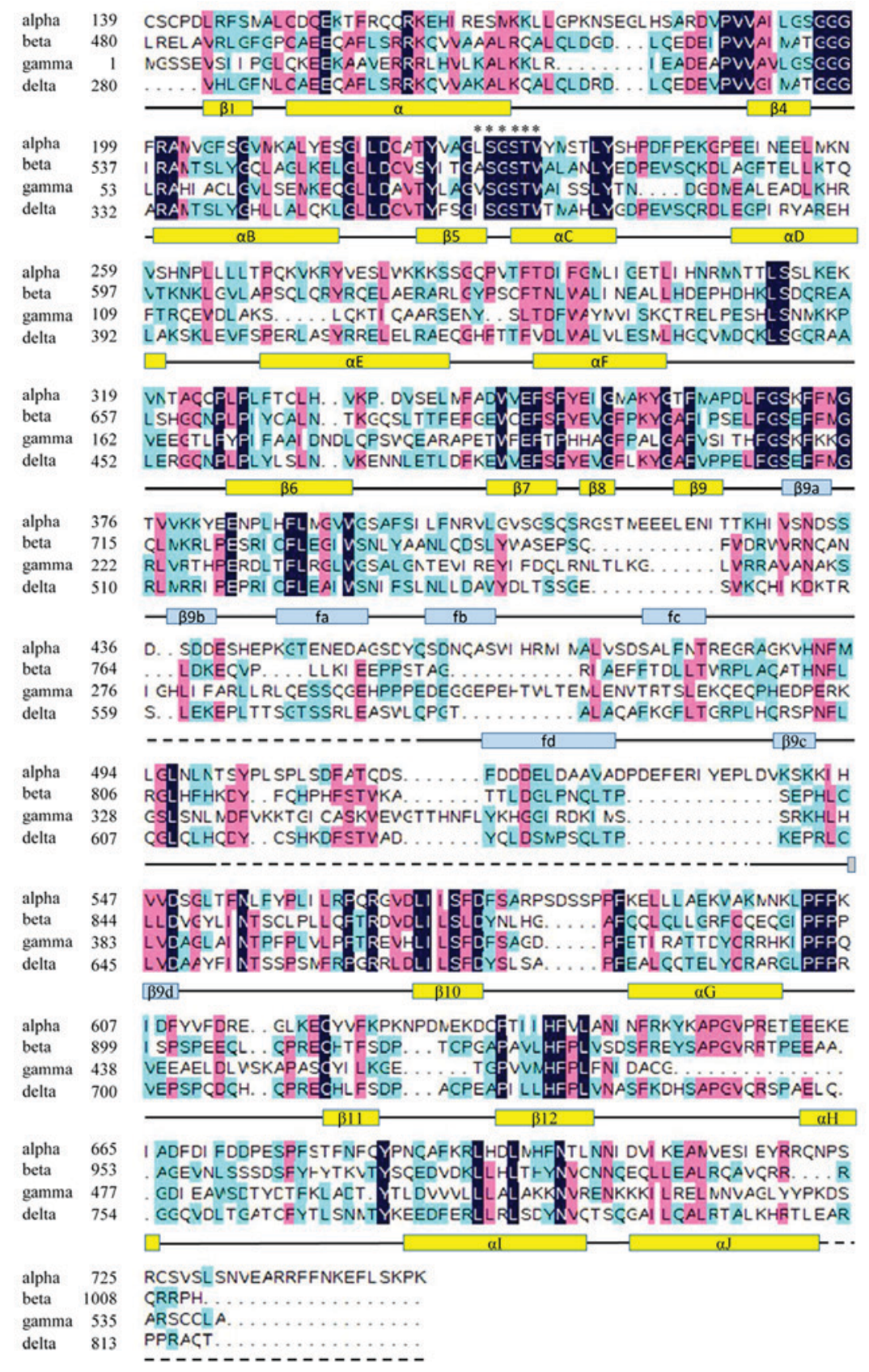

Figure 3. Primary structure alignment of cPLA2 $\alpha, \beta, \gamma$ and $\delta$. Plot of the secondary structure observed in the X-ray crystal structure of cPLA2 $\alpha$ is labeled below the sequences and the central core is colored yellow and purple. Residues indicated with dotted lines are not included in the X-ray structure. The asterisks identify residues lining the active site. cPLA2, cytosolic phospholipase A2.

observed between the risk of SPTB and PLA2G4C rs1366442 polymorphism $(\mathrm{P}>0.05)$.

Stratified analysis. Further stratified analysis was performed to assess whether the association between these SNPs and the risk of SPTB would be modified with specific participant's characteristics, such as maternal age, GDM status and neonatal sex (Table III). All associations of SNPs were independent of maternal age, GDM status and neonatal sex (all $\mathrm{P}>0.05$ ). There were no significant interactions between PLA2G4D rs4924618 and participant's characteristics on the risk of SPTB $(\mathrm{P}>0.05)$.

Three-dimensional structure of cPLA2 $\delta$. The SNP rs4924618 introduced a Ser-to-Thr point mutation at position 434 of
cPLA2 $\delta$, which encodes a polypeptide of 818 amino acids that display 30\% identity in its catalytic domain (amino acids 273 to 818 ) with three other lipases, cPLA $2 \alpha, \beta$ and $\gamma$. In order to elucidate the possible structural effect of this substitution, homology modeling was performed from the crystal structure of cPLA2 $\alpha$ to locate the mutation site (Fig. 1). A Ramachandran plot of main chain torsion angles indicated that most residues have a reasonable conformation (Fig. 2), demonstrating that the model is of good quality.

Fig. 1A demonstrated the constructed model superposed with the template. As expected, the tertiary structure of cPLA $2 \delta$ displayed considerable agreement with that of cPLA2 $\alpha$, especially in the $\alpha / \beta$ central core (yellow region in Figs. 1A and B), 
which consists of a 10 -stranded mixed $\beta$ sheet surrounded by $9 \alpha$ helices. This result was further verified by the multiple amino acid sequence alignment of all the four cPLA2 isozymes (Fig. 3), where the conserved residues are mainly concentrated within the core (26). The active site necessary for catalysis is conserved in cPLA2 $\delta$ at Ser361, following $\beta 5$ (Fig. 3). Similarly to the active site of cPLA $2 \alpha$, Ser361 is placed at the bottom of a deep, narrow active site funnel, which penetrates one-third of the way into the catalytic domain (Fig. 1B).

The mutation site Ser434 lies in the C-terminus of $\alpha$ helix $\mathrm{F}$ of the conserved central core, which is outside but in close to the bottom of the active site funnel spatially (14.92 A). Moreover, helix F, together with helices D, E and the interwoven loops, connect the region encompassing the catalytic site (the interconnecting loop between $\beta 5$ and helix $C$ ) with the further four $\beta$ strands of the core $(\beta 6-\beta 9)$.

\section{Discussion}

In the present study, the authors investigated whether maternal PLA2G4C and PLA2G4D gene polymorphisms modified the risk of SPTB in a Chinese population. The results indicated that the maternal AT/TT genotype (dominant model) of PLA2G4D rs4924618, but not PLA2G4C rs1366442 was associated with a reduced risk of SPTB. To the best of the authors' knowledge, the protective effect of PLA2G4D polymorphism on SPTB has not been reported before in any population.

PLA2G4D encodes the $\delta$ form of cPLA2, which is one of the key proteins involved in PGs synthesis $(19,27)$. PGs are well-known mediators in the inflammatory signaling pathways to accompany the switch of the myometrium from a quiescent to a contractile state (14). Increased expression and bioavailability of PGs have been observed in pregnant compared to non-pregnant women, in late compared to early gestation, suggesting a link between PGs and initial steps in parturition $(28,29)$. Indeed, fetal PG infusion was illustrated to provoke premature delivery of the ovine fetus (30). Clinically, PGs are used as a pharmacological labor inducer, and drugs inhibiting PG synthesis are effective in preventing preterm labor (14).

Based on the studies mentioned above, the authors concluded that the missense SNP rs4924618 may result in disadvantage for cPLA $2 \delta$ with regard to the expression, localization and/or activity. The homology modeling result indicated that rs4924618-associated position (Ser434) located at the conserved central core of the catalytic domain and was exposed on the protein surface (Fig. 1), demonstrating that even small changes at this site may not be tolerated. In addition, the proximity of Ser434 to the active site Ser361 prompted us to predict that the Ser434Thr mutation would cause perturbation in the physical conformation of the active site funnel to decrease the catalytic activity of cPLA $2 \delta$, downregulate the synthesis of PGs and, therefore, prolong the process of labor. The facts that human cPLA2 $\delta$ was tissue-specifically expressed in cervix (19) and murine cPLA2 $\delta$ was exclusively expressed in placenta (31) support the present speculation indirectly.

A case-control study involving diverse clinical populations showed minor A allele of PLA2G4C rs1366442 was detrimentally associated with PTB in the US Hispanic population (18).
However, for this PLA2G4C SNP analyzed in the present study, a null association was observed, which reminded us of considering the heterogeneity and population-specific effects among different ethnic cohorts. Possibly, this provided more evidence that differences in the genetic background of ethnic groups contribute to the variation in the rate of preterm birth among regions and countries.

Admittedly, the current study has several limitations. First, only a very limited number of SNPs from the PLA2G4C and PLA2G4D genes were analyzed. In addition, the sample size is relatively small, which may not be typical for the general population and has not enough power to examine the association of SNPs in early PTB ( $<34$ weeks of gestation). Although sample size is a limitation to study design, given the patterns of associations observed within genes and the functional analysis with the bioinformatics methods, it is clear that several of the findings are consistent with established literature. These results need to be confirmed by larger replication studies, particularly in another ethnic cohorts. More research, especially experiments using appropriate cell and animal models, are required to explain how the SNP rs4924618 influence the protein's structure and function during parturition progress.

In conclusion, the authors have presented that the polymorphism of PLA2G4D rs4924618 was associated with a reduced risk of SPTB in a Chinese population. Meanwhile, bioinformatics tools were used to investigate the potential SNP function. These results provide novel insights into the genetic contribution to the etiology of SPTB.

\section{Acknowledgments}

The authors would like to thank the pregnant women who have participated in the present study and all obstetric care providers who have assisted the authors in the implementation of the study. This work was supported by the grants from the Guangzhou Science and Technology Bureau (grant nos. 2011Y2-00025 and 2012J5100038), Guangdong Provincial Department of Science and Technology (grant no. 2014A020213022) and National Natural Science Foundation of China (grant no. 81673181).

\section{References}

1. Howson CP, Kinney MV, McDougall L and Lawn JE; Born Too Soon Preterm Birth Action Group: Born too soon: Preterm birth matters. Reprod Health 10 (Suppl 1): S1, 2013.

2. Goldenberg RL, Culhane JF, Iams JD and Romero R: Epidemiology and causes of preterm birth. Lancet 371: 75-84, 2008.

3. Moutquin JM: Classification and heterogeneity of preterm birth. BJOG 110 (Suppl 20): 30-33, 2003.

4. Bhattacharya S, Raja EA, Mirazo ER, Campbell DM, Lee AJ, Norman JE and Bhattacharya S: Inherited predisposition to spontaneous preterm delivery. Obstet Gynecol 115: 1125-1133, 2010.

5. Clausson B, Lichtenstein P and Cnattingius S: Genetic influence on birthweight and gestational length determined by studies in offspring of twins. BJOG 107: 375-381, 2000.

6. Romero R, Velez Edwards DR, Kusanovic JP, Hassan SS, Mazaki-Tovi S, Vaisbuch E, Kim CJ, Chaiworapongsa T, Pearce BD, Friel LA, et al: Identification of fetal and maternal single nucleotide polymorphisms in candidate genes that predispose to spontaneous preterm labor with intact membranes. Am J Obstet Gynecol 202: 431. e1-e34, 2010.

7. Velez DR, Fortunato SJ, Thorsen P, Lombardi SJ, Williams SM and Menon R: Preterm birth in Caucasians is associated with coagulation and inflammation pathway gene variants. PLoS One 3: e3283, 2008. 
8. Engel SA, Erichsen HC, Savitz DA, Thorp J, Chanock SJ and Olshan AF: Risk of spontaneous preterm birth is associated with common proinflammatory cytokine polymorphisms. Epidemiology 16: 469-477, 2005.

9. Simhan HN, Krohn MA, Roberts JM, Zeevi A and Caritis SN: Interleukin-6 promoter-174 polymorphism and spontaneous preterm birth. Am J Obstet Gynecol 189: 915-918, 2003.

10. Tsai HJ, Liu X, Mestan K, Yu Y, Zhang S, Fang Y, Pearson C, Ortiz K, Zuckerman B, Bauchner H, et al: Maternal cigarette smoking, metabolic gene polymorphisms, and preterm delivery: New insights on GxE interactions and pathogenic pathways. Hum Genet 123: 359-369, 2008.

11. Mann PC, Cooper ME, Ryckman KK, Comas B, Gili J, Crumley S, Bream EN, Byers HM, Piester T, Schaefer A, et al: Polymorphisms in the fetal progesterone receptor and a calcium-activated potassium channel isoform are associated with preterm birth in an Argentinian population. J Perinatol 33: 336-340, 2013.

12. Dolan SM, Hollegaard MV, Merialdi M, Betran AP, Allen T, Abelow C, Nace J, Lin BK, Khoury MJ, Ioannidis JP, et al: Synopsis of preterm birth genetic association studies: The preterm birth genetics knowledge base (PTBGene). Public Health Genomics 13: 514-523, 2010.

13. Sykes L, MacIntyre DA, Teoh TG and Bennett PR: Anti-inflammatory prostaglandins for the prevention of preterm labour. Reproduction 148: R29-R40, 2014.

14. Khan AH, Carson RJ and Nelson SM: Prostaglandins in labor-a translational approach. Front Biosci 13: 5794-5809, 2008.

15. Capper EA and Marshall LA: Mammalian phospholipases A(2): Mediators of inflammation, proliferation and apoptosis. Prog Lipid Res 40: 167-197, 2001.

16. Kita Y, Ohto T, Uozumi N and Shimizu T: Biochemical properties and pathophysiological roles of cytosolic phospholipase A2s. Biochim Biophys Acta 1761: 1317-1322, 2006.

17. Tithof PK, Roberts MP, Guan W, Elgayyar M and Godkin JD: Distinct phospholipase A2 enzymes regulate prostaglandin E2 and F2alpha production by bovine endometrial epithelial cells Reprod Biol Endocrinol 5: 16, 2007.

18. Plunkett J, Doniger S, Morgan T, Haataja R, Hallman M, Puttonen H, Menon R, Kuczynski E, Norwitz E, Snegovskikh V, et al: Primate-specific evolution of noncoding element insertion into PLA2G4C and human preterm birth. BMC Med Genomics 3: 62, 2010.

19. Chiba H, Michibata H, Wakimoto K, Seishima M, Kawasaki S, Okubo K, Mitsui H, Torii H and Imai Y: Cloning of a gene for a novel epithelium-specific cytosolic phospholipase A2, cPLA2delta, induced in psoriatic skin. J Biol Chem 279: 12890-12897, 2004
20. Tao R, Yu Y, Zhang X, Shi J, Guo Y, Wang C, Han B, Xu Q, Shang H, Zhang X, et al: A family based study of the genetic association between the PLA2G4D gene and schizophrenia. Prostaglandins Leukot Essent Fatty Acids 73: 419-422, 2005.

21. Ning LF, Yu YQ, GuoJi ET, Kou CG, Wu YH, Shi JP, Ai LZ and Yu Q: Meta-analysis of differentially expressed genes in autism based on gene expression data. Genet Mol Res 14: 2146-2155, 2015.

22. Vigod SN, Kurdyak PA, Dennis CL, Gruneir A, Newman A Seeman MV, Rochon PA, Anderson GM, Grigoriadis S and Ray JG: Maternal and newborn outcomes among women with schizophrenia: A retrospective population-based cohort study. BJOG 121: 566-574, 2014

23. Männistö T, Mendola P, Kiely M, O'Loughlin J, Werder E, Chen Z, Ehrenthal DB and Grantz KL: Maternal psychiatric disorders and risk of preterm birth. Ann Epidemiol 26: 14-20, 2016.

24. Biasini M, Bienert S, Waterhouse A, Arnold K, Studer G, Schmidt T, Kiefer F, Gallo Cassarino T, Bertoni M, Bordoli L and Schwede T: SWISS-MODEL: Modelling protein tertiary and quaternary structure using evolutionary information. Nucleic Acids Res 42: W252-W258, 2014.

25. Humphrey W, Dalke A and Schulten K: VMD: Visual molecular dynamics. J Mol Graph 14: 33-38, 1996.

26. Dessen A, Tang J, Schmidt H, Stahl M, Clark JD, Seehra J and Somers WS: Crystal structure of human cytosolic phospholipase A2 reveals a novel topology and catalytic mechanism. Cell 97: 349-360, 1999

27. Ghosh M, Tucker DE, Burchett SA and Leslie CC: Properties of the Group IV phospholipase A2 family. Prog Lipid Res 45: 487-510, 2006

28. Hayashi M, Inoue T, Hoshimoto K, Hirabayashi H, Negishi H and Ohkura T: The levels of five markers of hemostasis and endothelial status at different stages of normotensive pregnancy. Acta Obstet Gynecol Scand 81: 208-213, 2002.

29. Wang X, Zuckerman B, Kaufman G, Wise P, Hill M, Niu T, Ryan L, Wu D and Xu X: Molecular epidemiology of preterm delivery: Methodology and challenges. Paediatr Perinat Epidemiol 15 (Suppl 2): 63-77, 2001.

30. Young IR, Deayton JM, Hollingworth SA and Thorburn GD Continuous intrafetal infusion of prostaglandin E2 prematurely activates the hypothalamo-pituitary-adrenal axis and induces parturition in sheep. Endocrinology 137: 2424-2431, 1996.

31. Ohto T, Uozumi N, Hirabayashi T and Shimizu T: Identification of novel cytosolic phospholipase A(2)s, murine cPLA(2)\{delta\}, \{epsilon\}, and \{zeta\}, which form a gene cluster with cPLA(2) \{beta\}. J Biol Chem 280: 24576-24583, 2005. 